\section{Commentary: Absence of ventricular assist device-related risk for rejection after heart transplantation: Good news given the "new normal"}

\author{
James K. Kirklin, MD
}

Bakir and colleagues ${ }^{1}$ report a robust analysis of rejection following support with a continuous-flow (CF) left ventricular assist device (LVAD), which provides convincing evidence that the "LVAD experience" before heart transplantation does not induce a pro-rejection milieu. No difference in cellular- or antibody-mediated rejection frequency or severity was noted between LVAD-supported patients and those who received a primary heart transplant. Also noteworthy are the excellent outcomes among the authors' LVAD population undergoing heart transplantation.

As reported by these authors and others, the low incidence of sensitization with current CF LVADS stands in sharp contrast to historical pulsatile pumps, such as the HeartMate XVE device, which gained prominence as the first durable device approved by the US Food and Drug Administration for long-term implantation. During the era before 2008 (the first Food and Drug Administration approval of a CF LVAD), sensitization possibly stimulated by the textured blood interface of the $\mathrm{XVE}^{2}$ generated intense interest from the heart transplant community because of the perceived increased challenges of identifying suitable donors and because of the association between

From the Division of Cardiothoracic Surgery, Kirklin Institute for Research in Surgical Outcomes (KIRSO), and Department of Surgery, University of Alabama at Birmingham (UAB), Birmingham, Ala.

Disclosures: Dr Kirklin reported partial salary paid to his institution for his role as director of DCC for STS INTERMACS.

The Journal policy requires editors and reviewers to disclose conflicts of interest and to decline handling or reviewing manuscripts for which they may have a conflict of interest. The editors and reviewers of this article have no conflicts of interest.

Received for publication June 25, 2020; revisions received June 25, 2020; accepted for publication June 26, 2020; available ahead of print July 13, 2020.

Address for reprints: James K. Kirklin, MD, Division of Cardiothoracic Surgery, Kirklin Institute for Research in Surgical Outcomes (KIRSO), Department of Surgery, University of Alabama at Birmingham (UAB), ZRB 739, 703 19th St South, Birmingham, AL 35294 (E-mail: jkirklin@uabmc.edu).

J Thorac Cardiovasc Surg 2022;163:138-9

0022-5223/ $\$ 36.00$

Copyright $₫ 2020$ Published by Elsevier Inc. on behalf of The American Association for Thoracic Surgery

https://doi.org/10.1016/j.jtcvs.2020.06.108

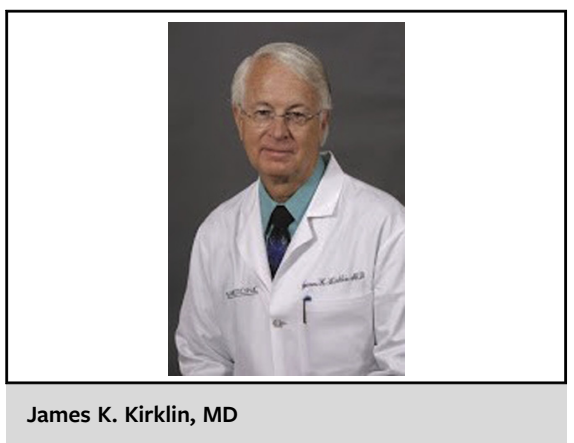

CENTRAL MESSAGE

Patients undergoing heart transplantation from continuous-flow ventricular assist devices do not experience increased rejection out to 2 years.

sensitization and worse rejection-related outcomes after transplant. ${ }^{3}$ Bakir and colleagues ${ }^{1}$ have demonstrated again that with current-era devices, such concerns have become largely neutralized. Of course, the finding of markedly reduced tendency for developing anti-HLA antibodies with CF LVADs is not new. The much lower incidence of antibody formation compared with the XVE was reported more than a decade ago. ${ }^{4}$

The similar low incidence of sensitization at transplant among those with and without LVAD support is important, given the much longer wait times among the patients with LVAD and the mean support time of more than a year. ${ }^{1}$ These findings will help reassure the LVAD/transplant teams of the relative safety (in terms of sensitization) of prolonged waitlist time for LVAD patients in this "new normal" of reduced priority of patients with LVADs under the current United Network of Organ Sharing allocation algorithm.

Another interesting observation was the absence of increased early or midterm rejection despite greater blood product use while waiting and during the transplant operation. Although this finding is encouraging, the known propensity for platelet transfusions (and less so other blood cell components) to induce anti-HLA antibody formation (especially Class I $)^{2}$ should remind us all about the importance of minimizing transfusions before transplantation whenever possible.

Thus, this report emphasizes the absence of major immunologic barriers in proceeding with heart transplantation from a CF LVAD. So, the conversation can return to the real Achilles heel of CF devices, namely adverse events 
during LVAD support. With the evolving device field, our focus is again directed toward solving the major barriers of stroke and other coagulation-related adverse events that challenge the longer-term device strategies, whether as permanent therapy or longer-term bridge to transplantation.

\section{References}

1. Bakir NH, Finnan MJ, MacGregor RM, Schilling JD, Ewald GA, Kotkar KD, et al. AATS 2020 cardiac allograft rejection in the current era of continuous flow left ventricular assist devices. J Thorac Cardiovasc Surg. 2022;163: 124-34.e8.

2. Colvin MM, Cook JL, Chang PP, Hsu DT, Kiernan MS, Kobashigawa JA et al. Sensitization in heart transplantation: emerging knowledge: a scientific statement from the American Heart Association. Circulation. 2019;139: e553-78.

3. Urban M, Gazdic T, Slimackova E, Pirk J, Szarszoi O, Maly J, et al. Alloimmunosensitization in left ventricular assist device recipients and impact on posttransplantation outcome. ASAIO J. 2012;58:554-61.

4. George I, Colley P, Russo MJ, Martens TP, Burke E, Oz MC, et al. Association of device surface and biomaterials with immunologic sensitization after mechanical support. J Thorac Cardiovasc Surg. 2008;135:1372-9. 